\title{
Rectal Perforation Associated with Magnetic Resonance-Guided Radiation Therapy (MRgRT) after Peri-SpaceOAR Abscess Formation: A Case Report.
}

Tairo Kashihara ( $\nabla$ kashiharatairo@gmail.com )

Kokuritsu Gan Center Chuo Byoin https://orcid.org/0000-0002-2471-605X

Koji Inaba

Kokuritsu Gan Kenkyu Center Chuo Byoin

Motokiyo Komiyama

Kokuritsu Gan Kenkyu Center Chuo Byoin

Hiroki Nakayama

Kokuritsu Gan Kenkyu Center Chuo Byoin

Kotaro lijima

Kokuritsu Gan Kenkyu Center Chuo Byoin

Shie Nishioka

Kokuritsu Gan Kenkyu Center Chuo Byoin

Hiroyuki Okamoto

Kokuritsu Gan Kenkyu Center Chuo Byoin

Nao Kikkawa

Kokuritsu Gan Kenkyu Center Chuo Byoin

Yuko Kubo

Kokuritsu Gan Kenkyu Center Chuo Byoin

Satoshi Shima

Kokuritsu Gan Kenkyu Center Chuo Byoin

Satoshi Nakamura

Kokuritsu Gan Kenkyu Center Chuo Byoin

Ayaka Takahashi

Kokuritsu Gan Kenkyu Center Chuo Byoin

Kana Takahashi

Kokuritsu Gan Kenkyu Center Chuo Byoin

\section{Kae Okuma}

Kokuritsu Gan Kenkyu Center Chuo Byoin

Naoya Murakami

Kokuritsu Gan Kenkyu Center Chuo Byoin

Hiroshi Igaki 
Kokuritsu Gan Kenkyu Center Chuo Byoin

\section{Yuko Nakayama}

Kokuritsu Gan Kenkyu Center Chuo Byoin

\section{Arinobu Fukunaga}

Kokuritsu Gan Kenkyu Center Chuo Byoin

\section{Yoshiyuki Matsui}

Kokuritsu Gan Kenkyu Center Chuo Byoin

\section{Hiroyuki Fujimoto}

Kokuritsu Gan Kenkyu Center Chuo Byoin

\section{Jun Itami}

Kokuritsu Gan Kenkyu Center Chuo Byoin

\section{Case report}

Keywords: Radiotherapy, Hydrogel spacer, Side effects, Hyperbaric oxygen therapy, MR-guided radiation therapy

Posted Date: July 27th, 2020

DOI: https://doi.org/10.21203/rs.3.rs-48536/v1

License: (c) (i) This work is licensed under a Creative Commons Attribution 4.0 International License. Read Full License 


\section{Abstract}

\section{Background}

In definitive radiation therapy for prostate cancer, SpaceOAR, a hydrogel spacer, is widely used to decrease irradiated dose and toxicity of rectum. On the other hand, periprostatic abscess formation is known as a rare adverse effect of SpaceOAR. There is a lack of reports clarifying the association between aggravation of abscesses and radiation therapy, and whether SpaceOAR insertion succeeded or not.

Case presentation

We report a case of a 78-year-old high-risk prostate cancer patient. After SpaceOAR insertion into the correct space, he started to receive external beam radiation therapy (EBRT). He developed a fever, perineal pain and frequent urination after the completion of EBRT, and the magnetic resonance imaging (MRI) revealed a peri-SpaceOAR abscess. Scheduled brachytherapy was postponed, administration of antibiotics and opioid via intravenous drip was commenced, and transperineal drainage was performed. After the alleviation of the abscess, additional EBRT instead of brachytherapy was performed with MRIguided radiation therapy (MRgRT). On the last day of the MRgRT, perineal pain reoccurred, and MRI and colonoscopy detected the rectal perforation. He received an intravenous antibiotics drip and hyperbaric oxygen therapy (HBOT), and fully recovered from the rectal perforation.

\section{Conclusions}

Our report indicates that EBRT can lead to severe rectum complication by causing inflammation for patients with a peri-SpaceOAR abscess. Furthermore, HBOT was effective for the peri-SpaceOAR abscess and rectal perforation associated with EBRT.

\section{Background}

A hydrogel spacer is used in radiation therapy (RT) for a variety of cancers to decrease the irradiated dose of organs at risk (OARs) [1-5]. Furthermore, in pelvic radiation therapy, a hydrogel spacer is used to decrease the rectum dose [6-9]. In definitive radiation therapy for prostate cancer, SpaceOAR, a hydrogel spacer, is widely used to decrease irradiated dose and toxicity of rectum [10-11]. A prospective randomized study revealed that insertion of SpaceOAR significantly reduced the rectum dose and toxicity, and improved bowel/urinary quality of life [12-14]. On the other hand, a patient who experienced periprostatic abscess formation after SpaceOAR insertion was reported [15]. In the preceding case report, it was not clear whether SpaceOAR insertion succeeded, although the infection improved after percutaneous drainage. Periprostatic abscess formation is a rare adverse effect of SpaceOAR, and the association between aggravation of abscesses and radiotherapy was not clarified. Herein, we present a case of rectal perforation following magnetic resonance-guided radiation therapy (MRgRT) after improvement of peri-SpaceOAR abscess. 


\section{Case Presentation}

The patient was a 78-year-old prostate cancer patient. He had no medical history other than surgery history of the goiter and nasal hemangioma. A prostate-specific antigen (PSA) level was $13.89 \mathrm{ng} / \mathrm{mL}$ in a routine evaluation. Clinical stage was T3a. An ultrasound-guided transperineal prostate biopsy was performed, and revealed Grade Group 4 adenocarcinoma in 1 of 24 specimens. Two months after the biopsy, administration of leutinizing hormone-releasing hormone (LHRH) agonist was initiated. He opted for external beam radiation therapy (EBRT) 46 Gy in 23 fractions combined with high-dose-rate (HDR) brachytherapy $15 \mathrm{~Gy}$ in 1 fraction as a definitive treatment. Four months after the initiation of hormone therapy, SpaceOAR was inserted into the space between the prostate and rectum and fiducial markers were inserted into prostate under local anesthesia with lidocaine (day 0 ). The insertions were completed without any side effects, and magnetic resonance imaging (MRI) confirmed that the SpaceOAR was inserted into the correct position (Fig. 1, left). Three weeks after the insertion of SpaceOAR (day 21), EBRT with computed tomographic (CT)-linac was initiated. Clinical target volume (CTV) was prostate, all seminal vesicles and whole pelvic lymph node regions. Planning target volume (PTV) margin of whole pelvis was $3 \mathrm{~mm}, 7 \mathrm{~mm}, 8 \mathrm{~mm}$ in the RL, SI, AP directions, respectively. Six days after the initiation of EBRT (day 27), he started to feel perineal pain. Owing to increased perineal pain, administration of antibiotics was initiated for a diagnosis of urinary tract infection on day 40 . Perineal pain gradually subsided, and he finished the oral antibiotics administration in one week (day 47). Four days after that (day 51), he felt perineal pain and frequent urination again, and restarted the antibiotic treatment. On day 60 , oral administration of opioid was initiated due to increasing perineal pain. The next day (day 61), he had a high fever, thus MRI was performed for detailed examination. Peri-SpaceOAR abscess was detected on the MRI (Fig. 1, middle), thus HDR brachytherapy was postponed, administration of antibiotics and opioid via intravenous drip started, and transperineal drainage was performed. The pain gradually improved and the abscess was found to have shrunk slightly on MRI, therefore administration of antibiotics changed to oral administration (day 76). Three weeks later (day 97), shrinkage of the abscess and improvement in inflammatory change were confirmed by MRI. Therefore, on day 112, an additional radiation therapy was initiated. At our conference, every other day EBRT of 20 Gy in 4 fractions was recommended as an additional radiation therapy instead of HDR monotherapy to decrease the dose per fraction. MRgRT with ${ }^{60} \mathrm{Co}$ MRIdian under a magnetic field of $0.345 \mathrm{~T}$ (ViewRay Inc.) was selected to assess intrafractional and interfractional motion of prostate, seminal vesicle and OARs such as rectum and bladder accurately. CTV was the prostate and seminal vesicles. PTV margin was $5 \mathrm{~mm}, 4 \mathrm{~mm}, 3 \mathrm{~mm}$ in the RL, SI, AP directions, respectively. On the last day of MRgRT (day 119), he experienced perineal pain again, and MRI was performed. On the MRI of day 120, peri-SpaceOAR inflammation aggravated and penetration to rectum was suspected (Fig. 1, right). Colonoscopy was subsequently performed, and penetration of SpaceOAR into the rectum was detected (Fig. 2). To treat rectal perforation, he was kept nothing per os and administration of antibiotics via intravenous drip and intravenous hyperalimentation started. Besides, he was transported to another hospital for receiving hyperbaric oxygen therapy (HBOT) (day 131). HBOT was initiated on day 131. After 24 times of HBOT for 5 weeks, the recovery from rectal 
perforation was confirmed by colonoscopy, and administration of antibiotics was seized. Ten weeks after the termination of HBOT, disappearance of a peri-SpaceOAR abscess was cofirmed on MRI (day 243).

\section{Discussion}

Radiotherapy is one of the most significant treatment modalities in prostate cancer [16-18]. In National Comprehensive Cancer Network (NCCN) guidelines, EBRT + androgen deprivation therapy (ADT) and EBRT + brachytherapy + ADT are options of the treatment of high-risk prostate cancer [19]. Our patient was scheduled to receive EBRT + HDR brachytherapy $+A D T$, however, the treatment plan was changed to EBRT + ADT because HDR brachytherapy could cause infection [20] and large dose per fraction could cause strong inflammation. Furthermore, MRgRT was selected due to its several potential advantages. In Murray $\mathrm{J}$ et al. [21] reported on 3 advantages; improvement in prostate visibility, monitor of intrafractional prostate position, daily adaptive re-planning. Owing to these advantages, the margin size of MRgRT in our patient was smaller than that of CT-based RT, as mentioned above.

Radiation-induced intestinal side effects such as bleeding and ulcer were sometimes observed [22-24], but rectal perforation associated with RT is rarely observed. On the contrary, a case of rectal ulceration due to insertion of SpaceOAR into the anterior rectal wall was reported by Teh AY et al. [25]. However, in this case, we confirmed by MRI, that SpaceOAR was inserted into the correct space between the prostate and rectum. Meanwhile, a periprostate abscess is a rare side effect of SpaceOAR [16]. In our paient, after improvement of the peri-SpaceOAR abscess, a rectal perforation was detected following EBRT. A rectal perforation would be caused by not only a peri-SpaceOAR abscess but also inflammation due to EBRT. To the best of our knowledge, this is the first report that a rectal perforation associated with a peri-SpaceOAR abscess in spite of insertion SpaceOAR into the correct position, and increased inflammation caused by EBRT was treated with HBOT.

HBOT has been reported to be effective for the treatment of an abscess [26-29]. Additionally, HBOT is also reported to be effective for the treatment of the side effects of radiation therapy [30-34]. We therefore recommend HBOT as the treatment for peri-SpaceOAR abscess and radiation-induced rectal perforation. In the management of radiation-induced hemorrhagic cystitis, early initiation of HBOT was reported to lead to better outcome [35]. In this previous report, HBOT within 6 months from hematuria onset resulted in a better response rate. In our patient, HBOT was initiated within 4 months from the onset of perineal pain, and within 2 weeks from the onset of rectal perforation. At the same time, it was feared that HBOT had a cancer-promoting effect and enhanced tumor progression. However, 3 reviews [36-39] reported that HBOT did not promote cancer growth; thus, the initiation of HBOT was a good treatment option.

\section{Conclusions}

Our report indicates that EBRT can lead to severe rectal complication by causing inflammation for patients with a peri-SpaceOAR abscess. Furthermore, HBOT was effective for the peri-SpaceOAR abscess 
and rectal perforation associated with EBRT.

\section{List Of Abbreviaions}

ADT, androgen deprivation therapy

$\mathrm{CT}$, computed tomographic

CTV, clinical target volume

EBRT, external beam radiation therapy

HBOT, hyperbaric oxygen therapy

HDR, high-dose-rate

LHRH, leutinizing hormone-releasing hormone

MRgRT, magnetic resonance imaging -guided radiation therapy

$\mathrm{MRI}$, magnetic resonance imaging

NCCN, National Comprehensive Cancer Network

OAR, organ at risk

PTV, planning target volume

PSA, prostate-specific antigen

$\mathrm{RT}$, radiation therapy

\section{Declarations}

\section{Ethics approval and consent to participate}

All analyses involving human participants performed in this study were approved by the institutional research committee (approval number, 2017-091) and were in accordance with the ethical standards of the committee and with the 1964 Helsinki declaration and its later amendments or comparable ethical standards.

\section{Consent for publication}

Consent for publication was obtained from the patient. 


\section{Availability of data and materials}

The datasets used and/or analyzed during the current study are available from the corresponding author on reasonable request.

\section{Competing Interests}

Dr. Inaba reports grants from Boston Scientific Japan, outside the submitted work.

Dr. Nakayama reports personal fees from AstraZeneca, outside the submitted work.

Dr. Igaki reports personal fees from Itochu, personal fees from ViewRay Inc., grants from HekaBio, outside the submitted work.

Dr. Itami reports grants and non-financial support from KeyJ, personal fees from Alpha Tau, personal fees from ItoChu, outside the submitted work.

\section{Funding}

This research received no specific grant from any funding agency in the public, commercial, or not-forprofit sectors.

\section{Authors' contributions}

All authors conceived of the study, and participated in its design and coordination and helped to draft the manuscript. They read and approved the final manuscript.

\section{Acknowledgements}

We would like to thank Editage (www.editage.jp) for English language editing.

\section{References}

1. Feng Z, Rao AD, Cheng Z, et al. Dose Prediction Model for Duodenum Sparing With a Biodegradable Hydrogel Spacer for Pancreatic Cancer Radiation Therapy. Int J Radiat Oncol Biol Phys. 2018 Nov 1;102(3):651-659.

2. Rao AD, Feng Z, Shin EJ, et al. A Novel Absorbable Radiopaque Hydrogel Spacer to Separate the Head of the Pancreas and Duodenum in Radiation Therapy for Pancreatic Cancer. Int J Radiat Oncol Biol Phys. 2017 Dec 1;99(5):1111-1120.

3. Kerdsirichairat T, Narang AK, Thompson E, et al. Feasibility of Using Hydrogel Spacers for BorderlineResectable and Locally Advanced Pancreatic Tumors. Gastroenterology. 2019 Oct;157(4):933-5. 
4. Struik GM, Pignol JP, Kolkman-Deurloo IK, et al. Subcutaneous spacer injection to reduce skin toxicity in breast brachytherapy: A pilot study on mastectomy specimens. Brachytherapy. 2019 Mar Apr;18(2):204-210.

5. Rao AD, Coquia S, De Jong R, et al. Effects of biodegradable hydrogel spacer injection on contralateral submandibular gland sparing in radiotherapy for head and neck cancers. Radiother Oncol. 2018 Jan;126(1):96-9.

6. Trifiletti DM, Garda AE, Showalter TN. Implanted spacer approaches for pelvic radiation therapy. Expert Rev Med Devices. 2016 Jul;13(7):633-40.

7. Kashihara T, Murakami N, Tselis N, et al. Hyaluronate gel injection for rectum dose reduction in gynecologic high-dose-rate brachytherapy: initial Japanese experience. J Radiat Res. 2019 Jul 1;60(4):501-508.

8. Murakami N, Shima S, Kashihara T, et al. Hyaluronic gel injection into the vesicovaginal septum for high-dose-rate brachytherapy of uterine cervical cancer: an effective approach for bladder dose reduction. J Contemp Brachytherapy. 2019 Feb;11(1):1-7.

9. Murakami N, Nakamura S, Kashihara T, et al. Hyaluronic acid gel injection in rectovaginal septum reduced incidence of rectal bleeding in brachytherapy for gynecological malignancies. Brachytherapy. 2020 Mar - Apr;19(2):154-161.

10. Hwang ME, Mayeda M, Liz M, et al. Stereotactic body radiotherapy with periprostatic hydrogel spacer for localized prostate cancer: toxicity profile and early oncologic outcomes. Radiat Oncol. 2019 Aug 2;14(1):136.

11. Wu SY, Boreta L, Wu A, et al. Improved rectal dosimetry with the use of SpaceOAR during high-doserate brachytherapy. Brachytherapy. 2018 Mar - Apr;17(2):259-264.

12. Mariados N, Sylvester J, Shah D, et al. Hydrogel Spacer Prospective Multicenter Randomized Controlled Pivotal Trial: Dosimetric and Clinical Effects of Perirectal Spacer Application in Men Undergoing Prostate Image Guided Intensity Modulated Radiation Therapy. Int J Radiat Oncol Biol Phys. 2015 Aug 1;92(5):971-977.

13. Hamstra DA, Mariados N, Sylvester J, et al. Continued Benefit to Rectal Separation for Prostate Radiation Therapy: Final Results of a Phase III Trial. Int J Radiat Oncol Biol Phys. 2017 Apr 1;97(5):976-985.

14. Shaikh T, Li T, Handorf EA, et al. Long-Term Patient-Reported Outcomes From a Phase 3 Randomized Prospective Trial of Conventional Versus Hypofractionated Radiation Therapy for Localized Prostate Cancer. Int J Radiat Oncol Biol Phys. 2017 Mar;15(4):722-31. 97(.

15. Hoe $\mathrm{V}$, Yao HH, Huang JG, et al. Abscess formation following hydrogel spacer for prostate cancer radiotherapy: a rare complication. BMJ Case Rep. 2019 Oct 5;12(10).

16. D'Amico AV, Whittington R, Malkowicz SB, et al. Biochemical Outcome After Radical Prostatectomy, External Beam Radiation Therapy, or Interstitial Radiation Therapy for Clinically Localized Prostate Cancer. JAMA. 1998;280(11):969-74. 
17. Parker CC, James ND, Brawley CD, et al. Radiotherapy to the Primary Tumour for Newly Diagnosed, Metastatic Prostate Cancer (STAMPEDE): A Randomised Controlled Phase 3 Trial. Lancet. 2018 Dec 1;392(10162):2353-2366.

18. Kashihara T, Nakamura S, Wakita A, et al. Importance of the site of positive surgical margin in salvage external beam radiation therapy for biochemical recurrence of prostate cancer after radical prostatectomy. Cancer Med. 2018 May;7(5):1723-30.

19. Mohler JL, Antonarakis ES, Armstrong AJ, et al. Prostate Cancer, Version 2.2019, NCCN Clinical Practice Guidelines in Oncology. J Natl Compr Canc Netw. 2019 May 1;17(5):479-505.

20. Emory CL, Montgomery CO, Potter BK, et al. Early complications of high-dose-rate brachytherapy in soft tissue sarcoma: a comparison with traditional external-beam radiotherapy. Clin Orthop Relat Res. 2012 Mar;470(3):751-8.

21. Murray J, Tree AC. Prostate cancer - Advantages and disadvantages of MR-guided RT. Clin Transl Radiat Oncol. 2019 Apr;1:18:68-73.

22. Fuccio L, Guido A, Andreyev HJ. Management of intestinal complications in patients with pelvic radiation disease. Clin Gastroenterol Hepatol. 2012 Dec;10(12):1326-34.e4.

23. Kashihara T, Murakami N, lizumi S, et al. Hemorrhage from Ascending Colon and Gluteal Muscle Associated with Sorafenib and Radiation Therapy: Radiation Dose Distribution Corresponded with Colonoscopy Findings and Computed Tomography Images. Pract Radiat Oncol. $2019 \mathrm{Jul}$ Aug;9(4):214-219.

24. Weiner JP, Wong AT, Schwartz D, et al. Endoscopic and non-endoscopic approaches for the management of radiation-induced rectal bleeding. World J Gastroenterol. 2016 Aug 21;22(31):697286.

25. Teh AY, Ko HT, Barr G, et al. Rectal ulcer associated with SpaceOAR hydrogel insertion during prostate brachytherapy. BMJ Case Rep. 2014 Dec 22;2014.

26. Sahin A, Kilic M, Dalgic N. A Case Report of a 4-Year-Old Boy with Intradural Spinal Cord Abscess Successfully Treated with Adjuvant Hyperbaric Oxygen Therapy. Turk Neurosurg. 2019;29(5):78992.

27. Kutlay M, Colak A, Yildiz S, et al. Stereotactic aspiration and antibiotic treatment combined with hyperbaric oxygen therapy in the management of bacterial brain abscesses. Neurosurgery. 2008 Feb;62(Suppl 2):540-6.

28. Cimşit M, Uzun G, Yildiz S. Hyperbaric oxygen therapy as an anti-infective agent. Expert Rev Anti Infect Ther. 2009 Oct;7(8):1015-26.

29. Ciodaro F, Gazia F, Galletti B, et al. Hyperbaric oxygen therapy in a case of cervical abscess extending to anterior mediastinum, with isolation of Prevotella corporis. BMJ Case Rep. 2019 Jul 10;12(7).

30. Hartmann A, Almeling M, Carl UM. Hyperbaric oxygenation (HBO) in the treatment of radiogenic side effects. Strahlenther Onkol. 1996 Dec;172(12):641-8.

31. Peusch-Dreyer $\mathrm{D}$, Dreyer $\mathrm{KH}$, Müller $\mathrm{CD}$, et al. Management of postoperative radiation injury of the urinary bladder by hyperbaric oxygen (HBO). Strahlenther Onkol. 1998 Nov;174(Suppl 3):99-100. 
32. Corman JM, McClure D, Pritchett R, et al. Treatment of radiation induced hemorrhagic cystitis with hyperbaric oxygen. J Urol. 2003 Jun;169(6):2200-2.

33. Cardinal J, Slade A, McFarland M, et al. Scoping Review and Meta-analysis of Hyperbaric Oxygen Therapy for Radiation-Induced Hemorrhagic Cystitis. Curr Urol Rep. 2018 Apr;13(6):38. 19(.

34. Ashamalla HL, Thom SR, Goldwein JW. Hyperbaric oxygen therapy for the treatment of radiationinduced sequelae in children. The University of Pennsylvania experience. Cancer. 1996 Jun 1;77(11):2407-12.

35. Chong KT, Hampson NB, Corman JM. Early hyperbaric oxygen therapy improves outcome for radiation-induced hemorrhagic cystitis. Urology. 2005 Apr;65(4):649-53.

36. Feldmeier J, Carl U, Hartmann K, et al. Hyperbaric oxygen: does it promote growth or recurrence of malignancy? Undersea Hyperb Med. 2003;30:1-18.

37. Daruwalla J, Christophi C. Hyperbaric oxygen therapy for malignancy: a review. World J Surg. 2006;30:2112-31.

38. Moen I, Stuhr LE. Hyperbaric oxygen therapy and cancer-a review. Target Oncol. 2012 Dec;7(4):23342.

\section{Figures}

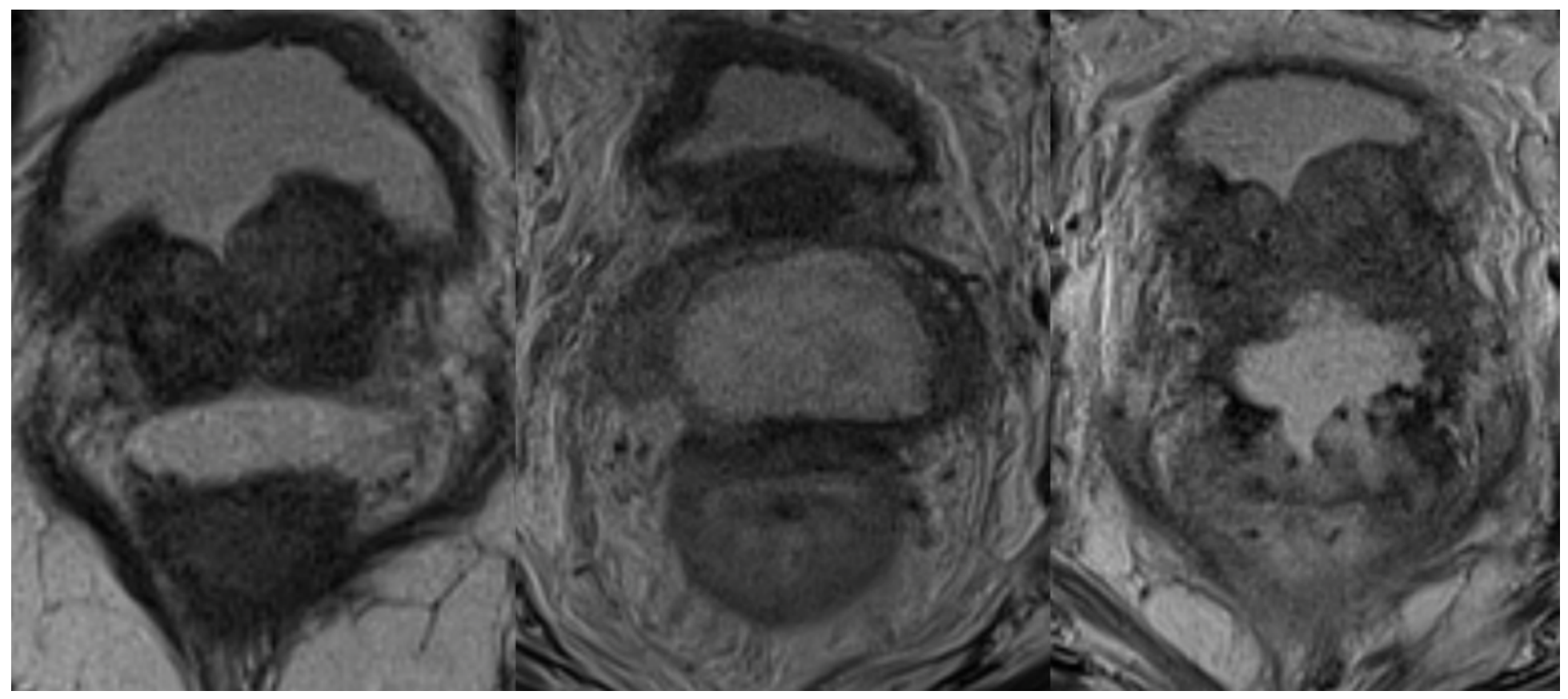

\section{Figure 1}

The changes of MRI findings after the SpaceOAR insertion. MRI taken one week after the SpaceOAR insertion (day 7, left). A peri-SpaceOAR abscess (day 61, middle) and a rectal perforation were detected on MRI (day 120, right). 


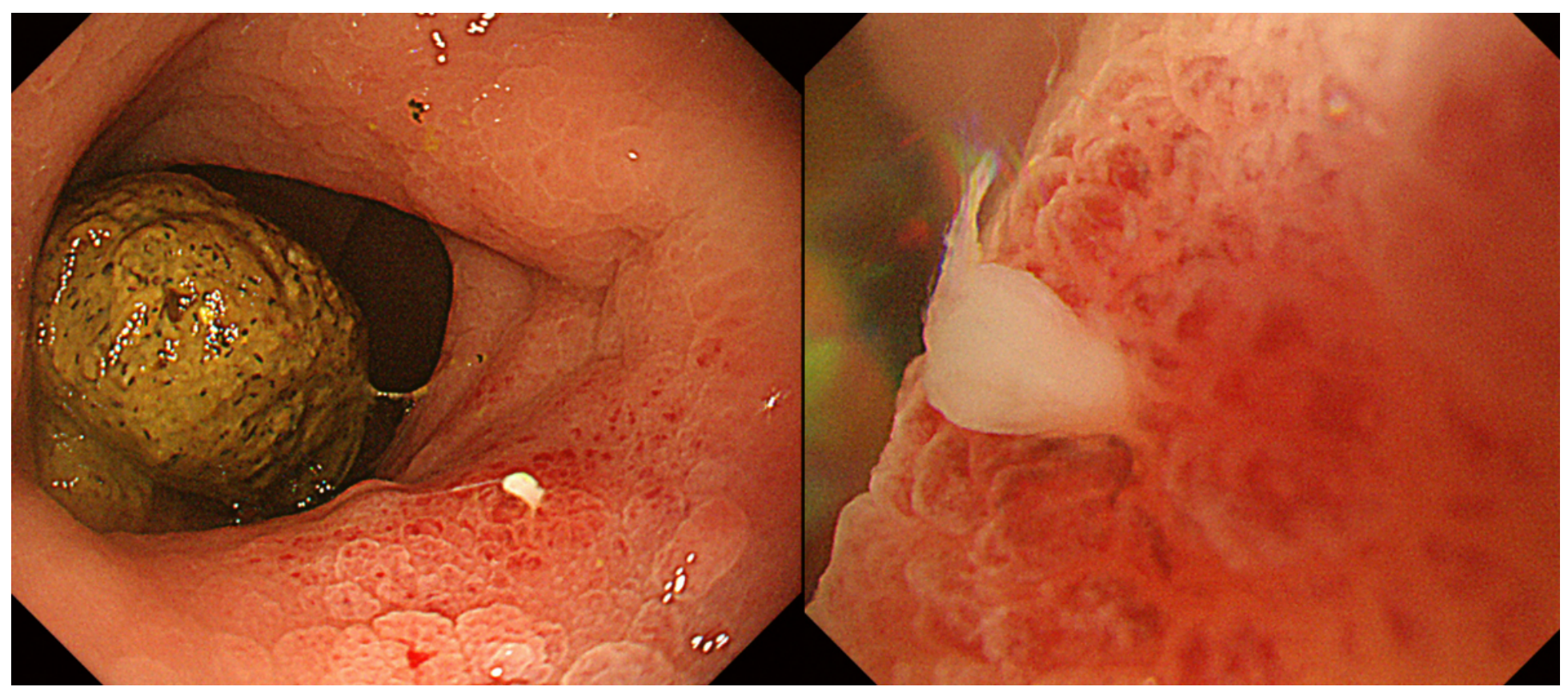

Figure 2

SpaceOAR penetration into rectum wall detected by colonoscopy. After the penetration of peri-SpaceOAR abscess into rectum wall was suspected on MRI, colonoscopy was performed. SpaceOAR penetrating rectum wall was detected on colonoscopy. 